\title{
DIMENSIONLESS ENGINEERING VARIABLES FOR MEASURING THE ITER AND REACTOR RELEVANCE OF TOKAMAK EXPERIMENTS
}

\author{
K.LACKNER \\ Max Planck Institut für Plasmaphysik - Euratom Association \\ D-85748 Garching, Germany
}

\begin{abstract}
$B_{t} \cdot a^{5 / 4}$ and $P_{\text {heat }} a^{3 / 4}$ are proposed as dimensionless engineering parameters to measure the reactor relevance of existing and planned confinement devices. These quantities - together with a density parameter that can be written as $n \cdot a^{3 / 4} / B_{t}$ - have to be conserved in plasma physics identity experiments on different size devices to respect the so-called Kadomtsev similarity constraints, and offer a coordinate system to map the approach to the reactor regime. Theoretical and semi-empirical models can be used in this coordinate space to produce isocontours for different dimensionless physics quantities, like the usual parameters $\rho *, v^{*} \beta$, but also for the intensity of collisional coupling, the excess of heating power over the L-H transition threshold, and the ratio of current redistribution to energy confinement time to visualize the distance to the regime of a fusion reactor.
\end{abstract}

\section{INTRODUCTION}

The discussion of the reactor relevance of existing experiments, the extrapolation to ITER, DEMO and commercial fusion reactors, and the dimensioning of possible future (ITERSatellite) tokamaks requires a judgement on the relative importance of size, magnetic field strength and heating power. The usual dimensionless plasma physics parameters ${ }^{1,2}$ : $\rho^{*}=\rho_{i} / a \propto \sqrt{T} /\left(a B_{t}\right), \quad v^{*}=a q / \lambda_{\text {mfp }} \propto a n_{e} q / T^{2}, \quad \beta_{t} \propto n_{e} T / B_{t}^{2}$, give a rigorously 
justifiable coordinate system for placing experiments but are only known after actual operation of the device. However, also engineering variables, fixed by the design and the provision of additional heating power can be brought into a dimensionless form, and can be specified prior to the actual operation of a device. We suggest a specific set of such parameters and argue that they define a suitable space for mapping the approach to reactor conditions.

\section{DIMENSIONLESS ENGINEERING VARIABLES}

Previous papers ${ }^{1,2,3}$ have outlined the requirements for "plasma physics identity experiments" between different devices. They regard, in addition to the identity of trivially dimensionless parameters fixing the plasma geometry and the poloidal/ toroidal field ratio $\left(\kappa, \delta, R_{o} / a, q ..\right)$, the prescription of heating power, magnetic field strength, density and plasma dimension so as to keep constant $B^{*} \sim B_{t} a^{5 / 4}, P^{*} \sim P_{\text {heat }} a^{3 / 4}, n a^{2}$, or combinations of these parameters. These parameters can be viewed as dimensionless, if we ignore the dimensions of natural constants, including the mass of the ion. In the following we show that such parameter combinations are also eminently useful to characterize the capabilities of existing and planned devices to contribute in scaling studies towards a fusion test facility or power reactor, and to suggest useful upgrades or even new facilities.

The above set is not unique - any product of powers of them would also qualify - but has the clear advantage of being linear in one of prime operation parameters, and to involve in addition, only the dimension of the device. The operational experience of tokamaks research shows, however, that they generally cannot exceed the so-called Greenwald/ Hugill/ Murakami density ${ }^{4}$, which - for $\kappa, \delta, R_{o} / a, q . .=$ const. - limits them to values $n \leq n_{G} \sim B_{t} / a$. 
For the purpose of outlining the operating regime of devices, this would therefore be the most relevant reference value, but does, unfortunately, not satisfy the dimensional constraints of plasma physics. We prefer therefore to use, instead, a dimensionally consistent parameter combination $n^{*} \sim n a^{3 / 4} / B_{t}$ closely approximating it as characteristic density ${ }^{3}$. Thermonuclear performance issues are to be explicitly excluded from this similarity, as nuclear reactions are not directly related to the plasma physics dimensionless parameters - as are atomic physics aspects determining, e.g. impurity behaviour, edge physics, NBI penetration ${ }^{3}$.

This set of parameters allows to delineate the operation space of geometrically similar devices in a 3-d plot, which can be reduced to 2-d if one assumes $n^{*} \approx$ const by comparing operation only at approximately similar Greenwald density. In this universal map of engineering design parameters the achievable plasma physics conditions: $\rho^{*}, v^{*}, \beta_{t}$ can be plotted as contours, but depend now, of course, on confinement law assumptions. For the ITER- $98_{\mathrm{y} 2}{ }^{5}$ scalingassumption these dimensionless physics parameters can be expressed as: $\rho^{*} \sim B^{*^{-0.75}} P^{*^{0.16}} n^{*^{-0.3}}, v^{*} \sim B^{*^{0.01}} P^{*^{-0.62}} n^{*^{2.18}}, \beta \sim B^{*^{-0.51}} P^{*^{0.31}} n^{*^{0.41}}$. Figure 1 shows in a log-log presentation the cut of these surfaces with the plane $n^{*}=$ const, together with a reference DEMO design point, and - as examples - the territory covered by the assumed useful operating ranges of a mid-size (ASDEX Upgrade), a large (JET) operating tokamak and of ITER. Table 1 shows the device parameters used in this and later figures.

Representations like fig. 1 do not bring revolutionary new insights, but rather illustrate in a more universal way the known limits to the simulation of reactor-regime plasma physics in smaller devices. While today's devices can readily attain - at least on the basis of their confinement, magnetic field and heating power constraints - the $\beta_{t}$ values of DEMO and ITER, they do so at significantly higher values of $\rho^{*}$. Even larger, however, are the 
differences in $v^{*}$, as - at given $n^{*}$ - present devices are much more collisional than ITER\&DEMO. (This is even more pronounced, if operation at the exact Greenwald density rather than its proxy $n^{*}$ - is considered, as will be shown in the last section.) A caveat concerns the relevant definition of collisionality: the parameter $v *$ used here is the one appearing in neoclassical theory. NTM physics depends on $v_{\text {drift }} \propto v^{*} / \rho^{*}$, which varies less strongly, and the coupling of electrons to ions is determined by the product of their collision frequency $v_{e i}$ with the energy confinement time $\tau_{E}$, discussed later.

The strength of this representation is that it allows comparing devices of very different size, as long as they are geometrically sufficiently similar (where geometrical similarity includes also the pitch angle of the magnetic field, and implies hence operation at the same $q_{95}$ ). Compact high field divertor-tokamaks (Alcator C-mod) can readily obtain similar values of $B^{*}$ as more conventional mid-size ones (DIII-D, ASDEX Upgrade), but would require also similar $P^{*}$ and hence higher heating power to achieve the same $\rho^{*}, v^{*}$ and $\beta$-values (Fig. 2). Constraints on access and problems of heat removal limit in practice actual high field devices to operation at lower $P_{\text {heat }}$ and hence much lower $P^{*}$. This could, in principle, however, be turned into an advantage for the study of the regimes with very high heat flux in the scrape-off layer, required by fusion reactor designs ${ }^{6}$. The reactor relevance of divertor physics and technology is determined by very different similarity considerations ${ }^{7,8}$ from those of the core plasma. As possibly relevant parameter combinations quoted to quantify these requirements, range from $P_{\text {heat }} / R$ to $P_{\text {heat }} / R^{2}$ and correspond to $\sim P^{*} / a^{7 / 4}$ to $\sim P^{*} / a^{13 / 4}$ respectively, a compact high field device at given $P^{*}, B^{*}, n^{*}$ and hence $\rho^{*}, v^{*}, \beta_{t}$ would operate at much more demanding divertor conditions. Whereas lower field devices would first encounter $\beta$ limits (or confinement degradation), a compact high field device provided with sufficient 
heating power could test reactor-like divertor conditions at dimensionless plasma physics parameters well within the established operating range of tokamaks.

Similar graphical representations can be used to compare device limitations concerning other properties, provided they can be formulated in a form consistent with the dimensionless Kadomtsev constraints ${ }^{1}$. Fig. 3 shows, in the same coordinate space and assuming again ITER98 $8_{\mathrm{y} 2}$ - confinement, that ITER will have a very similar coupling between electrons and ions (measured by $v_{e i} \tau_{E} \sim B^{* 1.75} n^{*^{2.30}} / P^{* 1.15}$ ) as present-day devices, that DEMO will be more comfortably above the $\mathrm{L} / \mathrm{H}$ power transition threshold (measured by $P_{\text {heat }} / P_{L H} \sim P^{*} /\left(B^{* 1.75} n^{* 0.75}\right)$, using the Kadomtsev-consistent version $P_{L H} \sim n_{e}^{0.75} B_{t} R^{2}$ of Ref. ${ }^{5}$ ) than ITER and that current equilibration on DEMO will take more energy confinement times than either on ITER or present devices (measured by $\tau_{\text {skin }} / \tau_{E} \sim P^{*^{1.15}} /\left(B^{*^{0.75}} n^{*^{1.3}}\right)$ ).

The above 3 landscapes (Figs.1-3) illustrate that a direct simulation of the ITER or DEMO parameter regime will not be possible in devices existing or under construction, in particular due to the incompatibility of the Greenwald density and the collisionality requirements. The extrapolation of results of ITER satellite devices will therefore have to rely strongly on improved theoretical understanding and modelling, and the separate satisfaction of (e.g.) the $v^{*}$ requirements on one, and the Greenwald density similarity on the other hand. A particular role should be played by multi-device, one-parameter scaling studies, in which two (if possibly theoretically justified) combinations of the 3 parameters $\rho^{*}, v^{*}, \beta_{t}$ are kept constant. The $P^{*}, B^{*}, n^{*}$ - presentation of the operating regime of devices, respectively 2-d projections of it, are also useful for outlining strategies for such scaling studies. Fig.4 shows, as examples, the projection of contours of constant $\left\{\beta, v^{*}\right\}$ or $\left\{\beta, v^{*} / \rho^{*}\right\}$ onto the $\left\{B_{t}^{*}, P^{*}\right\}$-plane, under 
the assumption of a gyro-Bohm confinement scaling $\tau_{G B} \sim B_{t}^{2} a^{3} / T^{3 / 2}$. In such a scan the normalized density $n *$ would also vary, increasing between and within each device in the direction of increasing $B_{t}^{*}, P^{*}$. Fig.4 shows that such scans would well be possible involving present mid-sized devices, devices of the JET (or JT-60SA) class, and ITER. At the same time it suggests to operate ITER partly also below its nominal field strength, to increase its flexibility for physics studies linking it to ITER-Satellites and extrapolating to DEMO. In fact, operation of ITER also at reduced heating power would then enable even dimensionless identity experiments between ITER and ITER-Satellite experiments, which have been shown to be a powerful tool to transfer operating scenarios from smaller to larger devices. A main practical obstacle to this would be the non-availability of suitable heating systems.

Specific strategies for a more physics based or more robust extrapolation of present day experiments to ITER have been proposed Waltz et al. ${ }^{9}$ and Barabaschi ${ }^{10}$. Also their strategies can be well illustrated using the maps of Figs.1-4. Waltz ${ }^{9}$ considers confinement scans at constant $\beta$ and $v^{*}$ to discriminate between different theoretical models for the dependence on $\rho^{*}$, and corresponds to the case considered in Fig.4. The actual scans require, of course, for every toroidal field (or current) setting a feedback adjustment of density and heating power (or a parametric search) to enforce the desired dimensionless parameter combination, and trace therefore a-priori unknown paths in the $B^{*}, P^{*}$ plane. Beforehand, however, the map of Fig.4 can already be applied to identify devices which can usefully participate in such scans, based on expectations from candidate confinement scalings (e.g. gyro-Bohm behaviour, as actually assumed for the lines in Fig.4, or ITER-98 y2 $_{2}$, and can hence serve also in the discussion of possible upgrades or new devices. 
The scaling strategy of Barabaschi ${ }^{10}$ suggest extrapolation to burning plasma experiments at constant $\beta$ and $n / n_{G R}$, and emphasizes - in accordance with our introductory remarks in section 2 - the need to respect geometrical similarity and identity of $q$. His scans correspond therefore to the $\beta=$ const. cases of Figs. 1,2, with the caveat that extrapolations should be based on the true Greenwald ratio $n / n_{G}$. As the latter does not exactly satisfy the Kadomtsev constraints, the projections of his extrapolations on the $B^{*}, P^{*}$-plane are not universal lines, but are slightly shifted, for each experiment, as illustrated by the bolder lines also shown in Fig.1.

\section{DISCUSSION}

The classification of devices by the two parameters $P^{*}, B^{*}$ (assuming that all of them can achieve similar $n^{*}$-values if impurities are adequately controlled) is justified only in case of strict geometrical similarity and constant value of $q$. In this case it does not matter whether $a$ or $R_{o}$ and $B_{t}$ or $I_{p} / a$ are used in the expressions for $P^{*}, B^{*}, n^{*}$. However, if a particular device - because e.g. of limits to the coil overturning forces - is not capable to reach low $q$ values at full toroidal field, the $B_{t}$-value compatible with the highest current appears more appropriate. This, for example was the reason to rate ASDEX Upgrade in table 1 at $2.5 T$, rather than its achievable maximum of $3.9 T$. Alternatively one might use $B^{*}=I_{p} a^{1 / 4}$ as suitable definition. It may be also argued that a quadratic rather than a linear expression in $B_{t}$ might be a better qualifier of experiments: the corresponding dimensionless variant of this would be $W^{*}=B^{*^{2}}=B_{t}^{2} a^{5 / 2}$ - indeed close to the magnetic energy - but this would not 
change anything in the relative placement of devices in Figs. 1 - 4, but only stretch the abscissa.

Heating methods and the associated fast particle physics partly introduce physics which is not covered by the above similarity arguments, as discussed in ref. ${ }^{3}$. ICRF-heating, at the proper frequency, in principle respects the approximations up to the point that devices at equal $P^{*}, B^{*}, n^{*}$ would also show the same behaviour of the non-thermal particles. Choosing, in NBI injection experiments, at given $P^{*}, B^{*}, n^{*}$, the beam energy like $\sim 1 / \sqrt{a}$ would also lead to a rigorously scaling slowing down population. The beam deposition, governed by atomic physics, however, would in general be different: e.g. for D-beam energies $>200 \mathrm{keV}$ the ratio of penetration depths to device size would scale like $\lambda_{\text {penetration }} / a \sim a^{5 / 8}$ among such an otherwise self-similar parameter set.

In discussing the capability of ITER satellite experiments also the pulse length capability is an important parameter. From the plasma physics side the most stringent requirement comes from the skin time, which - as used in Fig. 3 - in reality will depend on confinement, and is hence not a good "engineering quantity". We suggest using instead a nominal skin time, which would result from plasma operation at a fixed $\beta$-value and a fixed value of the Greenwald proxy $n^{*}$. A dimensionless pulse length would thus be defined like $\tau^{*}=\tau_{\text {pulse }} /\left(B^{3 / 2} a^{25 / 8}\right)$. Due to the $T^{3 / 2}$-dependence of the skin time, use of the "true" Greenwald limit (not respecting plasma physics similarity constraints) makes a significant difference in this case, leading to an alternative definition of $\tau^{*}=\tau_{\text {pulse }} /\left(B^{3 / 2} a^{7 / 2}\right)$. Normalizing the reference parameters $B^{3 / 2} a^{25 / 8}$ and $B^{3 / 2} a^{7 / 2}$ to a nominal pulse length of $500 \mathrm{~s}$ for ITER yields, for the maximum field values quoted in table 1, for JET 30 and $23 \mathrm{~s}$, for ASDEX Upgrade 2.2 and $1.3 \mathrm{~s}$ and for C-mod 1.1 and $0.5 \mathrm{~s}$, respectively, as equivalent 
pulse lengths. Referring technically feasible pulse durations to such reference parameters appears particularly useful for inertially cooled high field devices, which can operate over a wide range of field values, but with correspondingly varying pulse lengths.

Most of the comparisons in Figs. 1-4 were confined to cases of rigorous plasma physics similarity. The coordinate system $B^{*}, P^{*}$ remains, however, useful for classification of devices and operating conditions, even if deviations from Kadomtsev-similarity are to be allowed for. In this case, contour lines are, however, not universally relevant across different devices. This effect has been pointed out above and is illustrated in Fig.1, taking the example of the scaling paths proposed by Barabaschi.

The parameters $B^{*} \sim B_{t} a^{5 / 4}, P^{*} \sim P_{\text {heat }} a^{3 / 4}$ appear useful to measure the proximity of different devices to ITER and DEMO in terms of the parameters mentioned. It has to be stressed, however, that confinement and general performance of a device can depend sensitively also on parameters other than $\rho^{*}, v^{*}$ and $\beta$, or $B^{*}$ and $P^{*}$. In addition to $q$ and the plasma shape, these will include properties of the plasma heating method (including the associated momentum input and the resulting plasma rotation) and plasma wall interaction.

Acknowledgements:

The author acknowledges helpful comments from Hartmut Zohm and Francesco Romanelli. 


\section{REFERENCES}

${ }^{1}$ B.B. KADOMTSEV, Fiz. Plazmy, 1, 531 (1975)

2 J.W. CONNOR and J.B. TAYLOR, Nucl. Fusion, 17, 1047 (1977)

${ }^{3}$ K. LACKNER, Comments Plasma Phy. Contr. Fusion, 13, 163 (1990)

${ }^{4}$ M. GREENWALD et al., Nucl. Fusion, 28, 2199 (1988)

${ }^{5}$ ITER PHYSICS EXPERT GROUP ON CONFINEMENT et al., Nucl. Fusion, 39, 2175 (1999)

${ }^{6}$ K. LACKNER et al., J. Nucl. Mat., 307-311, 10 (2002),

${ }^{7}$ K. LACKNER, Comments Plasma Phy. Contr. Fusion,15, 359 (1994)

${ }^{8}$ L.H.HUTCHINSON and G.C. VLASES, Nucl. Fusion, 36, 783 (1996)

${ }^{9}$ R.E. WALTZ, J.C. DeBOO and M.N. ROSENBLUTH, Phys. Rev. Lett., 65,2390 (1990)

${ }^{10}$ P.BARABASCHI, Nucl. Fusion 41,155 (2001) 


\begin{tabular}{|l|l|l|l|l|l|}
\hline & $\mathrm{B}_{\mathrm{t}, \min }[\mathrm{T}]$ & $\mathrm{B}_{\mathrm{t}, \max }[\mathrm{T}]$ & $\mathrm{P}_{\text {heat,min }}[\mathrm{MW}]$ & $\mathrm{P}_{\text {heat,max }}[\mathrm{MW}]$ & $\mathrm{a}[\mathrm{m}]$ \\
\hline DEMO & 6 & 6 & 500 & 500 & 2.5 \\
\hline ITER & 2.6 & 5.2 & 73 & 150 & 2 \\
\hline JET & 1 & 3.5 & 5 & 40 & 1 \\
\hline ASDEX Upgrade & 1 & 2.5 & 2.5 & 25 & 0.5 \\
\hline DIII-D & 0.8 & 2.2 & 2.5 & 25 & 0.67 \\
\hline Alcator C-mod & 3 & 9 & 1.5 & 6 & 0.22 \\
\hline
\end{tabular}

TABLE 1: Device parameters used in Figs. 1-4 


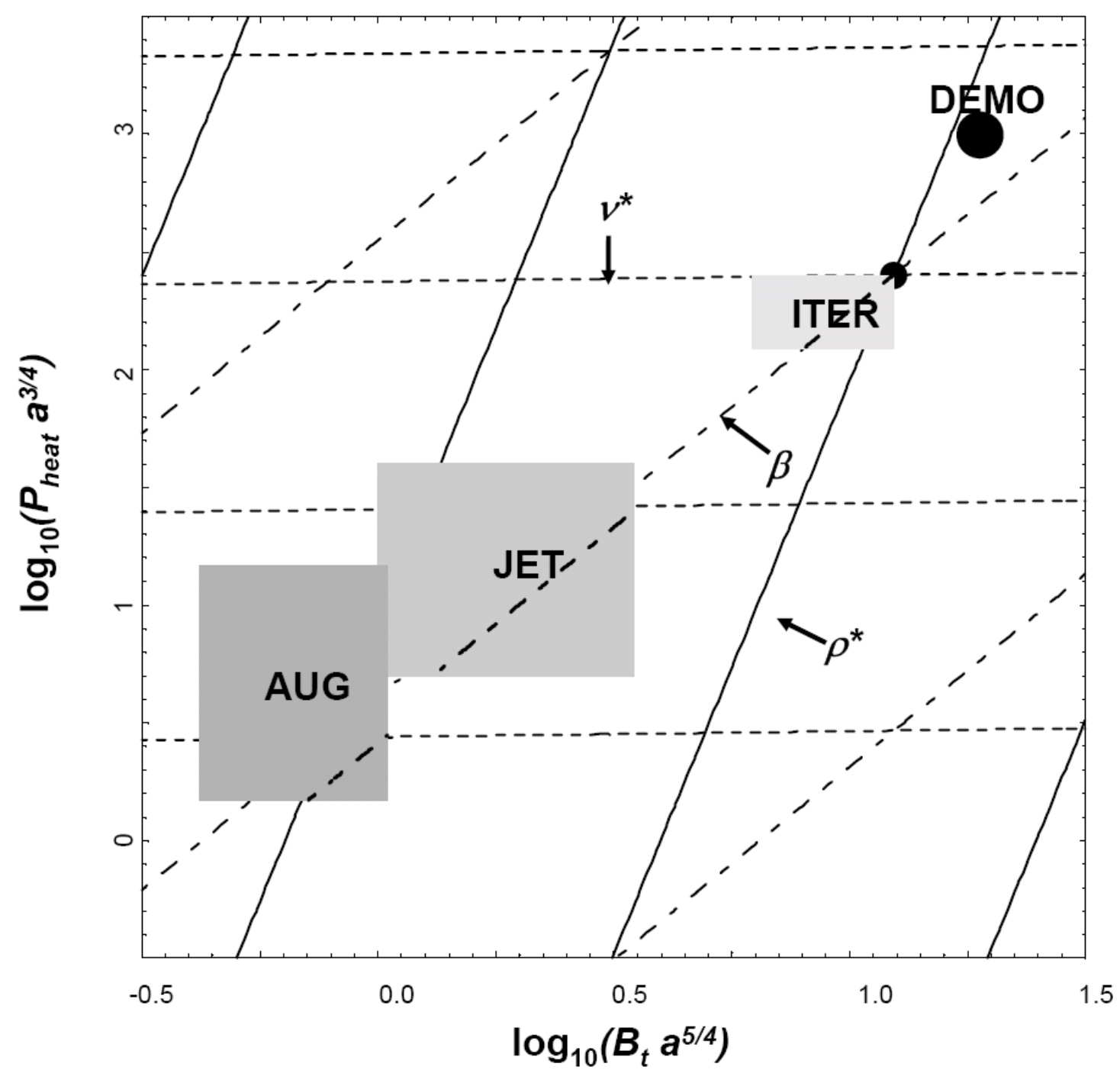

Fig.1: Attainable dimensionless plasma physics parameters at constant $n *$ under the ITER98y2 confinement law assumptions. Iso-contours of $v^{*}, \rho^{*}, \beta$ are spaced a factor 4 apart, with arrows indicating the directions of increasing parameter values. For ITER both the nominal operating parameters (dot) as well as an operating range described in Table 1 (shaded region) are indicated. For the nominal ITER- $\beta$ case, the impact of assuming $n / n_{G}=$ const. (rather than $n^{*}=$ const. ) is shown. In the latter case iso-contours depend in addition on absolute size of the device, so that only the segments corresponding to the respective device parameters of JET and ASDEX Upgrade are shown. 


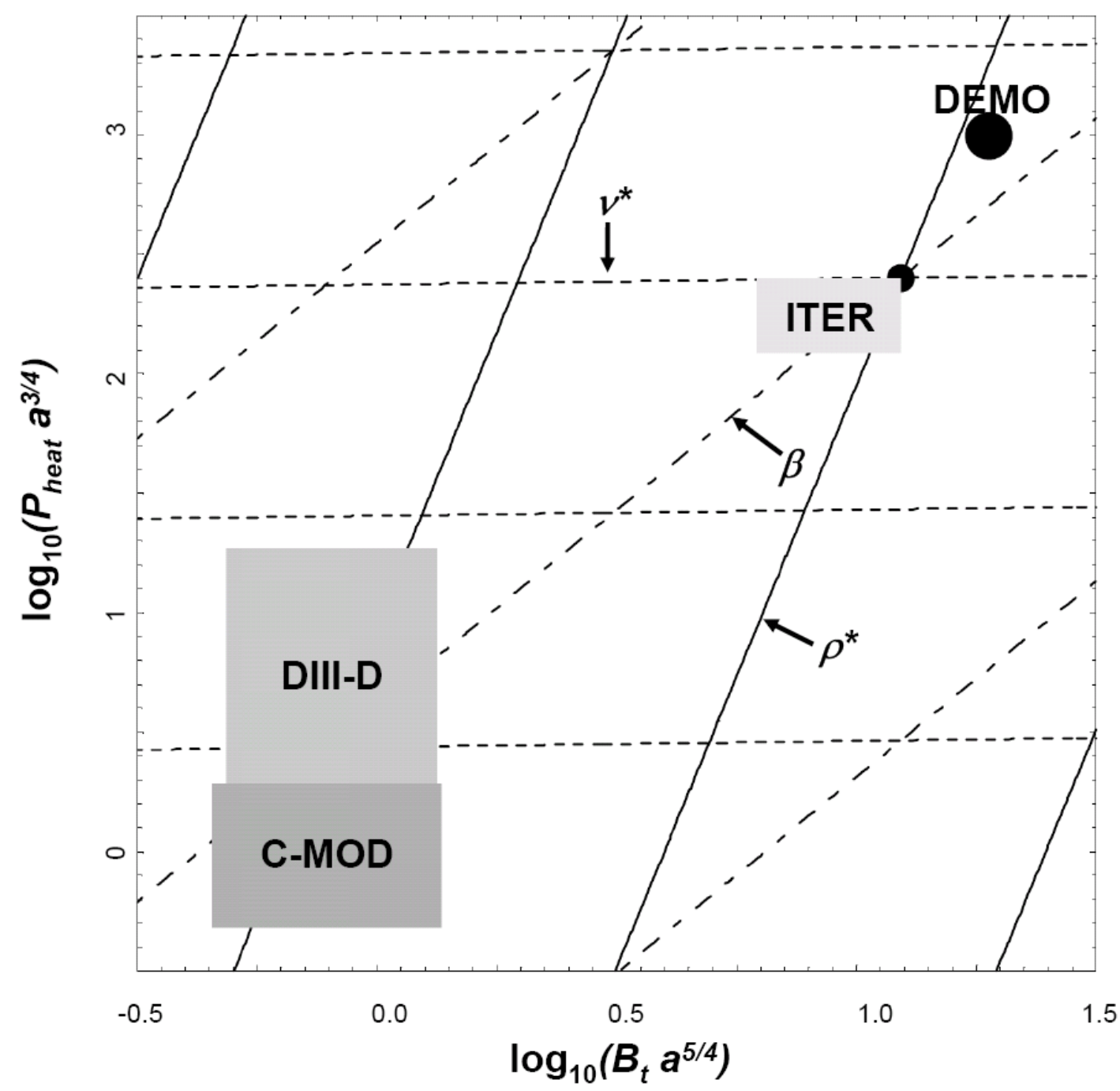

Fig.2 : As fig.1, but highlighting the difference in operation regime between existing high and medium field devices. 


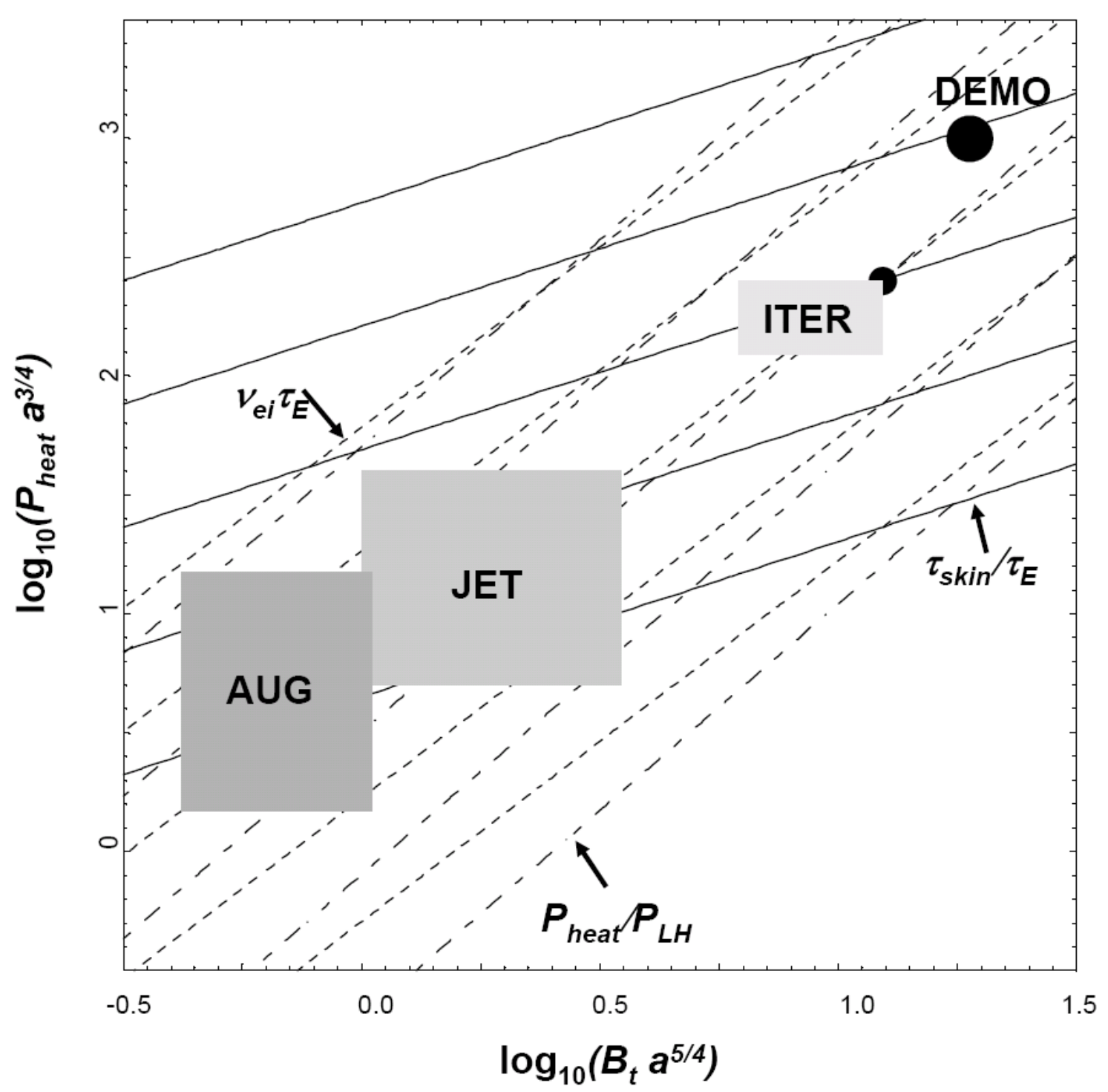

Fig.3: Trends in characteristic parameter ratios between existing devices, ITER and a possible DEMO. The ratios shown measure the collisional electron-ion coupling: $v_{e i} \tau_{E}$, the proximity to the $L / H$-transition threshold: $P_{h e a t} / P_{L H}$, and the ratio of skin to energy confinement time: $\tau_{\text {skin }} / \tau_{E}$. 


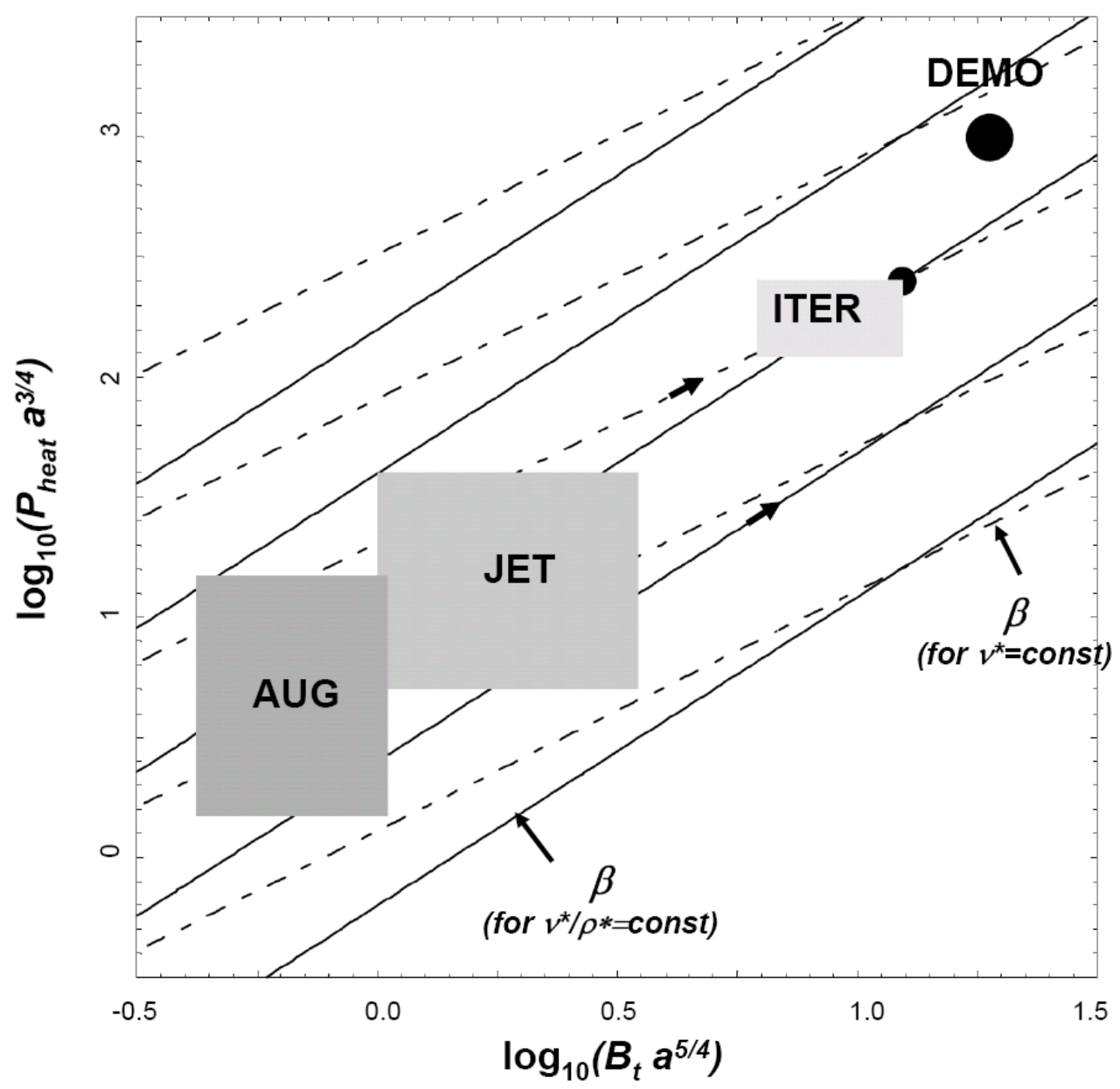

Fig. 4: Use of the $B_{t}^{*}, P^{*}$ landscape for mapping strategies for parameter scans involving different size devices, keeping two dimensionless parameters constant. In the case for this figure $n^{*}$ would be varied in a scan at constant $\left\{\beta, v^{*}\right\}$ or $\left\{\beta, v^{*} / \rho *\right\}$. 